\title{
Trifluoroacetyl-Activated Nitrogen-Nitrogen Bond Cleavage of Hydrazines by Samarium (II) lodide
}

\author{
Hui Ding, Gregory K. Friestad* \\ Department of Chemistry, University of Vermont, Burlington, VT \\ 05405 USA \\ gregory.friestad@uvm.edu
}

\section{Supporting Information}

Materials and Methods. Reactions employed oven- or flame-dried glassware under nitrogen unless otherwise noted. THF and toluene were distilled from sodium/benzophenone ketyl under argon. $\mathrm{CH}_{2} \mathrm{Cl}_{2}$ was distilled from $\mathrm{CaH}_{2}$ under argon or nitrogen. Nitrogen was passed successively through columns of anhydrous $\mathrm{CaSO}_{4}$ and R3-11 catalyst for removal of water and oxygen, respectively. Samarium iodide was prepared from diiodomethane and Sm powder in THF according to a literature procedure, ${ }^{1}$ but with one-third the amount of THF. The concentration of $\mathrm{SmI}_{2}$ was assumed to be $0.3 \mathrm{M}$ based on the preparation; the actual concentration is likely somewhat lower. All other materials were used as received or purified by standard procedures. Thin layer chromatography (TLC) employed glass $0.25 \mathrm{~mm}$ silica gel plates with UV indicator. Flash chromatography columns were packed with 230-400 mesh silica gel as a slurry in the initial elution solvent. Melting points are uncorrected. Nuclear magnetic resonance (NMR) data were obtained at operating frequencies of 500 $\mathrm{MHz}\left({ }^{1} \mathrm{H}\right)$ and $125 \mathrm{MHz}\left({ }^{13} \mathrm{C}\right)$. Infrared spectra were recorded using a single beam FT-IR spectrophotometer by standard transmission methods or by use of an attenuated total reflectance (ATR) probe. Optical rotations were determined using a digital polarimeter operating at ambient temperature. Low resolution mass spectra were obtained using sample introduction by dip, liquid chromatography or gas chromatography. Highresolution mass spectra were performed by University of Illinois at Urbana-Champaign (Urbana, IL). Combustion analyses were performed by Atlantic Microlab (Norcross, GA).

\footnotetext{
${ }^{1}$ Molander, G. A.; Kenny, C. J. Org. Chem. 1991, 56, 1439-1445.
} 
<smiles>C=CC[C@H](NN1C(=O)OC[C@H]1Br)c1ccc(OC)c(OC)c1</smiles>

(4S,1'S)-3-(1'-(3,4-Dimethoxyphenyl)but-3'-enylamino)-4-phenylmethyloxazolidin2-one (1b). ${ }^{2}[\alpha]_{\mathrm{D}}{ }^{23}-75\left(c 0.08, \mathrm{CHCl}_{3}\right)$. IR (film) 3286, 2934, 2835, 1753, 1593, 1516, 1455, 1262, 1237, 1141, $1092 \mathrm{~cm}^{-1} ;{ }^{1} \mathrm{H}$ NMR (500 MHz, $\left.\mathrm{CDCl}_{3}\right) \delta$ 7.25-7.18 (m, 7H), $6.83(\mathrm{~d}, J=8.1 \mathrm{~Hz}, 1 \mathrm{H}), 5.78$ (dddd, $J=17.3,7.2,7.2,7.2 \mathrm{~Hz}, 1 \mathrm{H}), 5.17(\mathrm{~d}, J=17.0 \mathrm{~Hz}$, $1 \mathrm{H}), 5.10(\mathrm{~d}, \mathrm{~J}=10.2 \mathrm{~Hz}, 1 \mathrm{H}), 4.45$ (br s, 1H), 4.24 (dd, J=7.1, 7.1 Hz, 1H), 3.88 (s, 3H), 3.86 (s, 3H), 3.80-3.79 (m, 2H), 3.21-3.15 (m, 2H), 2.51 (dd, J=13.1, 9.5 Hz, 1H), 2.46$2.43(\mathrm{~m}, 2 \mathrm{H}) ;{ }^{13} \mathrm{C} \mathrm{NMR}\left(125 \mathrm{MHz}, \mathrm{CDCl}_{3}\right) \delta 157.6,149.1,148.7,135.9,134.5,134.3$, 129.0, 128.8, 126.9, 120.3, 118.3, 111.1, 110.8, 65.8, 62.4, 58.1, 56.0, 55.9, 40.3, 36.8; MS (APCI) $\mathrm{m} / \mathrm{z}$ (relative intensity) $383\left([\mathrm{M}+\mathrm{H}]^{+}, 7 \%\right)$. Anal. Calcd for $\mathrm{C}_{22} \mathrm{H}_{26} \mathrm{~N}_{2} \mathrm{O}_{4}: \mathrm{C}$, 69.10; H, 6.85; N, 7.32. Found: C, 68.91; H, 6.75; N, 7.30.<smiles>O=C1CCCCN1NCc1ccccc1</smiles>

1-(Benzylamino)piperidin-2-one (1e). IR (film) 3271, 3029, 2945, 2868, 1637, 1457, 1412, $1162 \mathrm{~cm}^{-1} ;{ }^{1} \mathrm{H}$ NMR $\left(500 \mathrm{MHz}, \mathrm{CDCl}_{3}\right) \delta$ 7.36-7.24 (m, 5H), 5.69 (br s, 1H), 3.93 (s, 2H), 3.29 (t, J=6.0 Hz, 2H), 2.39 (t, J=6.0 Hz, 2H), 1.75-1.69 (m, 4H); ${ }^{13} \mathrm{C}$ NMR (125 $\left.\mathrm{MHz}, \mathrm{CDCl}_{3}\right) \delta 168.8,137.5,129.1,128.4,127.5,53.7,49.9,32.2,23.4,21.0$; MS (APCI) (relative intensity) $\mathrm{m} / \mathrm{z} 205\left([\mathrm{M}+\mathrm{H}]^{+}, 100 \%\right)$; HRMS-CI $(\mathrm{m} / \mathrm{z})[\mathrm{M}+\mathrm{H}]^{+}$calcd for $\mathrm{C}_{12} \mathrm{H}_{16} \mathrm{~N}_{2} \mathrm{O}$, 205.1341; found, 205.1343.<smiles>O=C1CCCN1NCc1ccccc1</smiles>

1-(Benzylamino)pyrrolidin-2-one (1f). IR (film) 3264, 3028, 2950, 2879, 1684, 1405, $1282 \mathrm{~cm}^{-1} ;{ }^{1} \mathrm{H}$ NMR $\left(500 \mathrm{MHz}, \mathrm{CDCl}_{3}\right) \delta 7.37-7.24(\mathrm{~m}, 5 \mathrm{H}), 4.95($ br s, $1 \mathrm{H}), 4.00(\mathrm{~s}$, $2 \mathrm{H}), 3.26(\mathrm{t}, J=7.5 \mathrm{~Hz}, 2 \mathrm{H}), 2.32\left(\mathrm{t}, J=8.0 \mathrm{~Hz}, 2 \mathrm{H}\right.$ ), 1.88 (quintet, $J=7.5 \mathrm{~Hz}, 2 \mathrm{H}$ ); ${ }^{13} \mathrm{C}$ NMR $\left(125 \mathrm{MHz}, \mathrm{CDCl}_{3}\right) \delta 173.1,137.5,128.9,128.5,127.6,54.0,48.3,29.2,16.3$; MS (APCI) $\mathrm{m} / \mathrm{z}$ (relative intensity) $191\left([\mathrm{M}+\mathrm{H}]^{+}, 100 \%\right)$; HRMS-CI $(\mathrm{m} / \mathrm{z})[\mathrm{M}+\mathrm{H}]^{+}$calcd for $\mathrm{C}_{11} \mathrm{H}_{14} \mathrm{~N}_{2} \mathrm{O}$, 191.1184; found, 119.1178 .

\footnotetext{
${ }^{2}$ Prepared by allylsilane addition: Friestad, G. K.; Ding, H. Angew. Chem. Int. Ed. 2001, 39, 4491-4493.
} 
<smiles>C=CCC(NC(=O)C(F)(F)F)c1ccccc1</smiles>

(S)- $\boldsymbol{N}$-(1'-Phenyl-but-3'-enyl)trifluoroacetamide (5a). mp 70-72 ${ }^{\circ} \mathrm{C} ;[\alpha]_{\mathrm{D}}^{25}-102(c 0.9$, $\mathrm{CHCl}_{3}$ ); IR (film) 3343, 3080, 2950, 2924, 2857, 1696, 1543, 1176, $926 \mathrm{~cm}^{-1} ;{ }^{1} \mathrm{H}$ NMR $\left(500 \mathrm{MHz}, \mathrm{CDCl}_{3}\right) \delta$ 7.37-7.24 (m, 5H), 6.57 (br, 1H), 5.67 (dddd, $J=17.5,10.5,7.5,7.5$ $\mathrm{Hz}, 1 \mathrm{H}), 5.17-5.12(\mathrm{~m}, 2 \mathrm{H}), 5.07$ (dddd, apparent quintet, $J=7.0 \mathrm{~Hz}, 1 \mathrm{H}), 2.64$ (dd, $J=6.9$, $6.9 \mathrm{~Hz}, 2 \mathrm{H}) ;{ }^{13} \mathrm{C}$ NMR $\left(125 \mathrm{MHz}, \mathrm{CDCl}_{3}\right) \delta 156.5\left(\mathrm{~d},{ }^{2} J_{\mathrm{CF}}=36.9 \mathrm{~Hz}\right), 139.5,132.8,128.9$, $128.1,126.4,119.3,115.9\left(\mathrm{~d},{ }^{1} J_{\mathrm{CF}}=286.4 \mathrm{~Hz}\right), 53.2,39.9$; MS (CI) $\mathrm{m} / \mathrm{z}$ (relative intensity) $244\left([\mathrm{M}+\mathrm{H}]^{+}, 3 \%\right)$. Anal. Calcd for $\mathrm{C}_{12} \mathrm{H}_{12} \mathrm{~F}_{3} \mathrm{NO}: \mathrm{C}, 59.26 ; \mathrm{H}, 4.97 ; \mathrm{N}, 5.76$. Found: $\mathrm{C}$, $59.24 ; \mathrm{H}, 4.94 ; \mathrm{N}, 5.61$.<smiles>C=CCC(NC(=O)C(F)(F)F)c1ccc(OC)c(OC)c1</smiles>

$\boldsymbol{N}$-(1-(3,4-Dimethoxyphenyl)-but-3-enyl)trifluoroacetamide (5b). (S)-5b: $[\alpha]_{\mathrm{D}}{ }^{25}-64(c$ $\left.0.17, \mathrm{CHCl}_{3}\right)$. This compound was spectroscopically $\left({ }^{1} \mathrm{H}\right.$ and ${ }^{13} \mathrm{C}$ NMR) identical to a racemic sample prepared by a different method. ${ }^{3}$ Racemic 5b: mp $54-56{ }^{\circ} \mathrm{C}$; IR (film) $3305,3082,2938,2839,1700,1518,1266,1183,1161,1027 \mathrm{~cm}^{-1}$; ${ }^{1} \mathrm{H}$ NMR (500 MHz, $\left.\mathrm{CDCl}_{3}\right) \delta$ 6.84-6.80 (m, 2H), $6.76(\mathrm{~s}, 1 \mathrm{H}), 6.45$ (br d, $\left.J=5.9 \mathrm{~Hz}, 1 \mathrm{H}\right), 5.67$ (dddd, $J=17.1$, $10.2,7.0,7.0 \mathrm{~Hz}, 1 \mathrm{H}), 5.17-5.11(\mathrm{~m}, 2 \mathrm{H}), 5.00$ (ddd, $J=7.1,7.1,7.1 \mathrm{~Hz}, 1 \mathrm{H}), 3.86$ (s, $3 \mathrm{H}), 3.85(\mathrm{~s}, 3 \mathrm{H}), 2.62(\mathrm{dd}, J=7.0,7.0 \mathrm{~Hz}, 2 \mathrm{H}) ;{ }^{13} \mathrm{C} \mathrm{NMR}\left(125 \mathrm{MHz}, \mathrm{CDCl}_{3}\right) \delta 156.4(\mathrm{~d}$, $\left.J_{\mathrm{CF}}=36.9 \mathrm{~Hz}\right), 149.4,149.0,133.0,132.0,119.0,118.5,115.9\left(\mathrm{~d}, J_{\mathrm{CF}}=286.5 \mathrm{~Hz}\right), 111.5$, $110.2,56.0(2 \mathrm{C}), 53.1,39.7 ;{ }^{13} \mathrm{C}$ NMR $\left(125 \mathrm{MHz}\right.$, toluene-d $\left.{ }_{8}\right) \delta 156.0\left(\mathrm{~d}, J_{\mathrm{CF}}=46.4 \mathrm{~Hz}\right)$, $150.1,149.7,133.5,132.3,118.5,118.1,116.4\left(\mathrm{~d}, J_{\mathrm{CF}}=285.3 \mathrm{~Hz}\right), 112.2,111.1,55.4$, 55.3, 53.0, 39.3;MS (APCI) $\mathrm{m} / \mathrm{z}$ (relative intensity) $304\left([\mathrm{M}+\mathrm{H}]^{+}, 6 \%\right)$. Anal. Calcd for $\mathrm{C}_{14} \mathrm{H}_{16} \mathrm{~F}_{3} \mathrm{NO}_{3}$ : C, 55.44; H, 5.32; N, 4.62. Found: C, 55.55; H, 5.36; N, 4.70.<smiles>CCC(NC(=O)C(F)(F)F)C(C)C</smiles>

(R)- $\mathrm{N}$-(1-Ethyl-2-methylpropyl)trifluoroacetamide (5c). ${ }^{4} \mathrm{mp} 46-47^{\circ} \mathrm{C} ;[\alpha]_{\mathrm{D}}{ }^{25}+13(c$ $0.22, \mathrm{CHCl}_{3}$ ). IR (film) 3301, 3101, 2908, 2883, 1700, 1557, 1400, 1185, $1157 \mathrm{~cm}^{-1} ;{ }^{1} \mathrm{H}$ NMR $\left(500 \mathrm{MHz}, \mathrm{CDCl}_{3}\right) \delta 5.90$ (br s, $\left.1 \mathrm{H}\right), 3.74$ (dddd, $J=10.0,10.0,5.5,5.5 \mathrm{~Hz}, 1 \mathrm{H}$ ), 1.84-1.76 (m, 1H), 1.69-1.61 (m, 1H), 1.43-1.34 (m, 1H), 0.92-0.88 (m, 9H); ${ }^{13} \mathrm{C}$ NMR $\left(125 \mathrm{MHz}, \mathrm{CDCl}_{3}\right) \delta 157.2\left(\mathrm{~d},{ }^{2} J_{\mathrm{CF}}=35.5 \mathrm{~Hz}\right), 116.1\left(\mathrm{~d},{ }^{l} J_{\mathrm{CF}}=286.4 \mathrm{~Hz}\right), 57.0,31.4,29.7$, 19.2, 17.6, 10.3.

\footnotetext{
${ }^{3}$ Hirabayashi, R.; Ogawa, C.; Sugiura, M.; Kobayashi, S. J. Am. Chem. Soc. 2001, 123, 9493-9499.

${ }^{4}$ Jin, Z.; Jin, H.L. Chromatographia 1994, 38, $22-28$.
} 
<smiles>CC(C)C(NC(=O)C(F)(F)F)c1ccccc1</smiles>

(R)- $N$-(2-Methyl-1-phenylpropyl)trifluoroacetamide (5d). This sample was $50.4 \%$ ee by HPLC analysis (Chiralcel AD, 99:1 hexane:isopropanol). mp 91-93 ${ }^{\circ} \mathrm{C}$; $[\alpha]_{\mathrm{D}}{ }^{24}+114(c$ 0.91, $\mathrm{CHCl}_{3}$ ); IR (film) 3318, 3085, 2966, 2868, 1698, 1556, 1207, 1172, $902 \mathrm{~cm}^{-1} ;{ }^{1} \mathrm{H}$ NMR (500 MHz, $\mathrm{CDCl}_{3}$ ) $\delta$ 7.36-7.19 (m, 5H), 6.58 (br s, 1H), 4.72 (dd, J=8.7, $8.7 \mathrm{~Hz}$, $1 \mathrm{H}), 2.17-2.09(\mathrm{~m}, 1 \mathrm{H}), 0.99(\mathrm{~d}, J=6.7 \mathrm{~Hz}, 3 \mathrm{H}), 0.83(\mathrm{~d}, J=6.7 \mathrm{~Hz}, 3 \mathrm{H}) ;{ }^{13} \mathrm{C}$ NMR $(125$ $\left.\mathrm{MHz}, \mathrm{CDCl}_{3}\right) \delta 156.6\left(\mathrm{~d},{ }^{2} J_{\mathrm{CF}}=36.5 \mathrm{~Hz}\right), 139.5,128.8,127.9,126.9,116.0\left(\mathrm{~d},{ }^{1} J_{\mathrm{CF}}=286.5\right.$ $\mathrm{Hz}$ ), 60.2, 33.2, 19.6, 18.8; MS (MALDI) $\mathrm{m} / \mathrm{z}$ (relative intensity) $245\left(\mathrm{M}^{+}, 7 \%\right)$. Anal. Calcd for $\mathrm{C}_{12} \mathrm{H}_{14} \mathrm{~F}_{3} \mathrm{NO}$ : C, 58.77; H, 5.75; N, 5.71. Found: C, 58.83; H, 5.73; N, 5.71.<smiles>C=CC[C@H](N)c1ccc(OC)c(OC)c1</smiles>

(S)-1-(3,4-Dimethoxyphenyl)but-3-enylamine (7). mp 55-56 ${ }^{\circ} \mathrm{C} ;[\alpha]_{\mathrm{D}}{ }^{23}-13.6(c 0.5$, $\mathrm{CHCl}_{3}$ ); IR (film) 3374, 3069, 2933, 2834, 1677, 1592, 1514, 1462, 1417, 1262, 1232, $1139,1028 \mathrm{~cm}^{-1}$; ${ }^{1} \mathrm{H}$ NMR $\left(500 \mathrm{MHz}, \mathrm{CDCl}_{3}\right) \delta$ 6.90-6.80 (m, 3H), 5.74 (dddd, $J=18.1$, $10.2,8.0,6.2 \mathrm{~Hz}, 1 \mathrm{H}), 5.08$ (dd, J=17.0, $1.3 \mathrm{~Hz}, 1 \mathrm{H}), 5.04$ (ddd, J=11.1, 1.5, $1.5 \mathrm{~Hz}, 1 \mathrm{H}$ ), 3.95-3.88 (m, 1H), 3.88 (s, 3H), 3.85 (s, 3H), 2.44-2.41 (m, 1H), 2.34-2.30 (m, 1H), 1.65 (br s, $2 \mathrm{H}) ;{ }^{13} \mathrm{C}$ NMR $\left(125 \mathrm{MHz}, \mathrm{CDCl}_{3}\right) \delta 149.1,148.1,138.6,135.6,118.4,117.5,111.1$, 109.6, 55.9, 55.1, 44.4, 38.6; MS (CI) $\mathrm{m} / \mathrm{z}$ (relative intensity) $208(\mathrm{M}+1,6 \%)$. Anal. Calcd for $\mathrm{C}_{12} \mathrm{H}_{17} \mathrm{~F}_{3} \mathrm{NO}_{2}$ : C, 69.54; H, 8.27; N, 6.76. Found: C, 68.94; H, 8.26; N, 6.57. 
Assessment of enantiomeric purity of 5b by HPLC analysis

HPLC analysis conditions: Chiralcel AD, 99:1 hexane:isopropanol, $1 \mathrm{~mL} / \mathrm{min}$.

(Upper, racemic 5b, Lower: $(\boldsymbol{S})-\mathbf{5 b}$.)
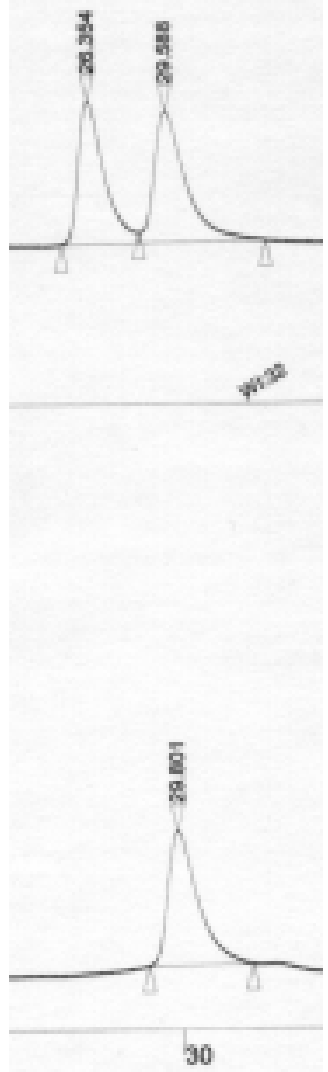